\title{
La ripartizione dell' energia fra le sesse di un lago
}

\author{
(The energy distribution among the seiches of a lake)
}

\author{
(i. Pezzoti (*)
}

Ricevulo il 5 Marzo 1975

\begin{abstract}
Riassure. - In questa nota è stato preso in esame in maniera approssimata il problema della ripartizione dell'energia fra le oscillazioni libere di un lango di diversa norlalita.

li ovvio che condizioni particolari possono inluire sull insorgere prevalente di ma sessa piuttosto che di unaltra, tultavia, statisticamente, la probabilita che si osservi l'uninodale is generalmente magriore di quella relativa allosservazione della binodale ece., perehé, sempre statisticamente, somo in media maggiori le ampiezze delleuninorlale rispetto alle altre.

la determinazione della ripartizione statistica delle energie, e quindi delle ampiezze, is lo seopo del presente lavoro.
\end{abstract}

Sumsar. - This work studies by approximation the problem of the energy distribution among the seiches of a lake of various node.

It is obvious that particular conditions may affect the prevalent rise of one seiche rather than another, however, statistically the probability to observe the uninodale is generally bigerer than the probability of observing the binodale ete.; becaluse, still statistically the amplitudes of the mninodale are. on the average, higger. compared with the others.

The determination of the statistical distribution of the energies and consequently the amplitudes. is the purpose of this work.

A complemento di precedenti indagini, mi sono proposto col presente lavoro, di esaminare, sia pure in morlo approssimato, il modo di ripartirsi clellenergia totale tra le sesse di varie nodalita presenti in un lago o bacino, sia esso naturale che artificiale, el anche in mari in cui si instamrino oscillazioni libere.

(*) Istituto di Ldraliea e Costruzioni Idrauliche. Politecnico, Torino. 
Isa ripartizione differenziata dellenergia alle vario frequenze fa sì, come è ben noto dalle osservazioni dirette, ehe la sessa minodale, ad esempio, sia, in un dato lago, a parita di altre conclizioni, ben più evidente della binodale, e questa della trinodale e così via.

Le ipotesi che generalmento si famno nelle teorie idlodinamiche delle sesse possono cosi riassumersi: lunghi bacini (canali) di largheza e profondità variabili; componente laterale del movimento orizzontale, normale alla linea di valle, trascurabile, cio ahe presuppone il moto orizzontale verificarsi soltanto parallelamente all'asse $x$, che, in corrispondenza della linea di valle, sta nel piano orizzontale costituito dalla superficie libera del lago in quiete. Queste ipotesi si ritengono praticamente soddisfatte se non si verificano brusche variazioni né in larghezza e profondità, né lungo la linea di valle.

Per onde lunghe, rispetto alla lungheza d'oncla delle quali la profondita $h$ del bacino sia piceola, laceeleratone verticale si ritiene. trascurabile. Conseguenza di questa ipotesi è che soltanto la pressione idrostatica risulta dinamicamente efficace; sono quindi da considerare soltanto le variazioni di pressione derivanti dalle variazioni di livello. Saturalmente, anche gli spostamenti orizontali $\xi$ somo nguali per tutte le particelle di ma stessa sezione trasversale $S(x)$.

Per dislivelli $\eta$ piccoli rispetto alle profondita $h$, l'equazione del movimento in un bacino generico assume la forma

$$
\frac{)^{2} \xi}{\partial t^{2}}=-\eta \frac{\partial \eta}{\partial x}
$$

L'equazione di continutì invece, se $b(x)$ indica la larghezza variabile del bacino misurata alla superficie libera, si scrive:

$$
\eta=-\frac{1}{h(x)} \cdot \frac{\partial}{\partial x} \cdot|\xi \cdot s(x)|
$$

Ia soluzione di queste equazioni conchuce all un problema ai limiti del 2o ordine che notoriamente ha soluzioni soltanto per determinati autovalori di un parametro; nel nostro aso, questi valoui sono i periodi delle possibili oscillazioni libere.

G. Chrystal $(1,3,8)$ trasforma le equazioni [1] e [2], che mediante lintroduzione delle variabili

$$
\|=\xi \cdot N(x)
$$




$$
v=\int_{0}^{x} b(x) \mathrm{dl} . x
$$

forniscono

$$
\frac{\partial^{2} \xi}{\partial t^{2}}=g \frac{\partial}{\partial x}\left\lfloor\frac{1}{b(x)} \frac{\partial\{S(x) \cdot \xi\}}{\partial x}\right\rfloor
$$

per eni moltiplicando per $S(x)$ e ponendo

$$
S(x) \cdot b(x)=\sigma(v)
$$

dove $x$ risulta, per la [4], funzione di $v$, si ottengono le equazioni del moto e di continuità sotio la forma:

$$
\left\{\begin{array}{l}
\frac{\partial-u}{\partial t-}=g \sigma(v) \frac{\partial^{2} u}{\partial v^{2}} \\
\eta=\cdots \frac{\partial u}{\partial v}
\end{array}\right.
$$

Se ora $u$ i rappresentabile merliante la somma di nua serie di semplici funzioni amoniche di $t$ clel tipo

$$
u=\sum_{I}^{\infty} U_{n}(v) \sin ()_{n}\left(t-t_{n}\right), \quad\left(\omega_{n}=\frac{\varepsilon \pi}{I_{n}}\right)
$$

tale da lapplesentare un'oscillazione stazionaria della massa, l'equazione di chrystal assume la forma:

$$
\sigma(v) \frac{d-U}{d v^{2}}+\frac{4 \pi^{2}}{y L^{-}} \cdot U=0
$$

avendo soppresso l'indice $n$ relativo all'ordine rlella sessa.

La curva rappresentiata dalla funzione $\sigma(v)$ è detta da Chrystal curva normale de] bacino d'acqua; la [7], con le posizioni

$$
\frac{b}{a}=z, \quad \frac{+\pi^{2} a^{2}}{g T^{2}}=\lambda
$$

dove a i la superficie totale rlel lago e gli alturi simboli noti e consueti, diviene

$$
\frac{d^{2} U}{d z^{2}}+\frac{\lambda U}{\sigma(z)}=0
$$


da integrarsi con le condizioni al contorno

$$
L(0)=U(1)=0
$$

Il problema è stato ampiamente trattato e risolto in varia guisa, sia numericamente che con metorli di tipo variazionale; tutti portano, sia pure con una certa complicazione nei calcoli, alla determinazione di valori $T_{n}$ dei periodi delle varie sesse.

Ia conoscenza dei valori suindicati non è peró quella che qui ci interessa direttamente, anche se i valori stessi sono fondamentali ai fini della riceror.

Il fine che ci proponiamo di ragginngere è quello di una valutazione, sia pure di massima, della ripartizione dell'energia fra le sesse di varia nodaliti e delle ampiezze posserlute dalle medesime, a pariti di sollecitazioni indotte nella massa liquida; mi sembra in effetti che l'argomento in questione non sia generalmente considerato dagli Autori che si sono finora oceupati di simili questioni.

E noto che unonda stazionaria puó ritenersi provocata dalla sovrapposizione di due onde di egual lunghezza che si propagano in sensi opposti; considerazioni di carattere meccanico e statistico, hanno conclotto Neumann, Pierson e altri $\left({ }^{4,5,6}\right)$ a stabilire per la variazione $\Delta E$ di energia medla, compresa in una lungheza d'onda, relativa ad una gamma di periodi $\Delta T$, per onde dirette, la relazione:

$$
\Delta E=K T+e^{-2\left(\frac{u T}{V}\right)^{2}} \Delta T
$$

¿love $\alpha=\frac{g}{2 \pi}, K$ è una costante cla determinarsi sperimentalmente e $r$ la velocita del vento che provoca le onde stesse; la [11] ì riferita naturalmente all'unitia di lunghezar di fronte d'onda piano e vale nell'ipotesi che la causa perturbante agisca sul mezzo liquido in modo aleatorio.

Dato che le sesse sono quasi sempre provocate da variazioni di pressione atmosferica, e quindi da venti, sfrutteremo l'ulteriore risultato di Neumann ehe per le onde molto lunghe assegna una relazione di proporzionalita fra $r$ e $T$, ed in particolare

$$
V \simeq \frac{g}{2 \pi} \sqrt{\frac{2}{3}} T
$$

Con la [12], la [11] diviene, indicando con $K^{\prime}$ una ulteriore costanto:

$$
\Delta E^{\prime}=K^{\prime} T^{1} \Delta T^{\prime}
$$


da cui considerando $\Delta E$ e $\Delta T$ al limite come differenziali e integrando, si ottiene per l'energia totale, per lunghezza d'onda e per unità di lunghezza di fronte un legame di proporzionalità tra $E$ e $T^{5}$; vale a dire

$$
E=K^{\prime \prime} T^{5}
$$

Il rapporto fra l'energia afferente alla sessa di norlalitì $n$ e quella posseduta dall'uninorlale sarà quindi:

$$
\frac{E_{n}}{E_{1}}=\left(\frac{T_{n}}{T_{1}}\right)^{5}
$$

e di conseguenza poiché l'energia totale media di un'onda progressiva compresa nel dominio anzirletto (per l'onda stazionaria cambierebbe solo un fattore numerico), vale:

$$
E=\frac{\hat{r}^{2} ?}{3}
$$

dove $\gamma$ è il peso specifico del liquirlo, $\lambda$ la lunghezza dell'onta e a la sua ampiezza, la [15] diviene

$$
\left(\frac{T_{n}}{T_{1}}\right)^{\bar{j}}=\frac{a_{n}{ }^{2} \lambda_{n}}{a^{2}{ }_{1} \lambda_{1}}
$$

e poiché $\lambda_{n}=c T_{n}$ e $c$ è pressoché costante per ogni onda, trattandosi di onde molto lunghe rispetto alla profonditì $(c=\sqrt{g h)}$, la [17] risulta

$$
\frac{a_{n}}{a_{1}}=\left(\frac{T_{n}}{T_{1}}\right)^{\prime}
$$

Ise [15] e [18] forniscono i rapporti di energia e di ampiezza fra la sessa di ordine n.mo e quella di ordine 1, tuttavia Chrystal ha dimostrato che la [9], nel caso di curva normale $\sigma(z)$ data da una porzione di curva quartica rel tipo

$$
\sigma(z)=\sigma_{0}\left(1 \mp z^{2}\right)^{2}
$$

è integrabile, e fornisce per il periodo $T_{n}$, il valore

$$
T_{n}=\frac{T_{0}}{1 n^{2}+\varepsilon}
$$

dove $T_{0}$ ed $\varepsilon$ sono due costanti proprie del lago (o del golfo) oscillante od $\varepsilon \quad 0$ a seconda che il segno fra parentesi nella [19] ̀̀ negativo o positivo. 
La [0], pur essendo approssimata, e non essendo in generale possibile dedurre $T_{0}$ ed $\varepsilon$ dalle costanti del sistema studiato, consente pero di valutare $T_{0}$ ed $\varepsilon$ quando siano noti, arl esempio, i valori di $T_{1}$ e $T_{2}$ e permette di conseguenza di valutare $T K$ con $K>2$.

Introducenclo la [20] nella [18] è possibile scrivere immediatamente

$$
\frac{a_{n}}{a_{1}}=\frac{1+\varepsilon}{n^{2}+\varepsilon}
$$

da cui si deduce, sia pure in via approssimata, che una sola costante, per ogni sistema liquirlo oseillante a pelo libero, è responsabile della distribuzione di ampiezze, e quindi di energia, fra le sesse di varia norlalitì.

Non ̀̀ facile, in genere, disporre di serie di ampiezze massime di sesse, dovute a cause aleatorie, e che non siano esaltate da particolari effetti di risonanza ece. Molto spesso i dati sperimentali sono ottenuti da limnografi posti in luoghi che si rivelano buoni per captare sesse di assegnata nodalita, ma pessimi per sesse di nodalita diversa, ragion per cui è difficile e molto impreciso risalire alle ampiezze massime.

Per le maree dell'Alto Arbiatico, da un recentissimo studio di P. Caloi (2) abbiamo derlotto i valori più attendibili per le prime 3 sesse; si ha:

$$
\left\{\begin{array}{l}
T_{1}=22^{\mathrm{n}} 40^{\mathrm{m}} \\
T_{2}=11^{\mathrm{n}} 40^{\mathrm{m}} \\
T_{\mathrm{a}}=7^{n} 30^{\mathrm{m}}
\end{array}\right.
$$

di consegnenza la costante caratiteristica $\varepsilon$ del mare, dal confronto frat [18] e [21] risulta

$$
\varepsilon \simeq 0,085 \pm 8
$$

Dall'altra nota ricerca di $\mathrm{S}$. Polli $\left({ }^{7}\right)$ relativa allo stesso mare, ho tratto le ampiezze massime delle stesse sesse:

$$
\begin{aligned}
& a_{1}=0,94 \mathrm{~m} \\
& a_{2}=0,20 " \text { rilevate il giorno } 28 \cdot 1 \cdot 1948 \\
& a_{3}=0,12 "
\end{aligned}
$$


Il confronto fra i valori $\frac{a_{n}}{a_{1}}$ teorici e sperimentali sono riportati nella tabella 1

'TABELAa l

\begin{tabular}{|c|c|c|}
\hline Rapporto & Teorico & Sperimentale \\
\hline$a_{2} / a_{1}$ & 0,2656 & 0,2127 \\
$a_{3} / a_{1}$ & 0,1194 & 0,1276 \\
\hline
\end{tabular}

nella quale è possibile osservare un soddisfacente accordo fra le misure e le determinazioni analitiche.

Occorre tuttavia molto maggior materiale sperimentale, ottenuto nelle ipotesi fatte, per poter confermare la validita rlelle [18] e [21].

\section{BIBI,IUCRAFIA}

(1) Calor P., 1948. - Le sesse del lago di Garda. "Annali di Geofisica "I.

(2) Canor P., 1973. - Sulle cause delle acque "alte " nell'Adriatico settentrionale con particolare riguardo alla laguna veneta. "Annali di Greofisica ", XXVI.

(3) CunYstai, G., 1905-06-07-08. - On the hydrodynamical theory of seiches Transactions of the Royal Society of Edinburgh, XLI, XLV, XLVI.

(4) Neumaxx (4., 1953. - On ocean wave spectra and nete method of forecasting wind-generated waves. Beach Erosion Board. 'Techn. Memo., 143.

(5) Plersox W. J., 1952. - An unified mathematical theory for the analysis, propagation and refraction of storm-generated surjace waves. $\mathrm{N}$. $\mathrm{Y}$. Univer. College of Engineering Dep. of Meteor., New York.

(6) Piersox W. J., Neumaxy (4. e Jayes R. W., 1955. - Practical methods for observing and forecasting Ocean Waves by means of wave spectra and statisties. II. O. Pnb. no 603, U. S. Navy Hydrogr. Off.

(`) Pold S., 1958. - Le sesse nellddriatico. "Annali di Geofisica ", X.

(8) Supiso G., 1965. - Le reti idrauliche. Ed. Patron, Bologna. 